\title{
Instability of Coulomb phase in QCD
}

\author{
Manuel Asorey* and Alessandro Santagata \\ Departamento de Física Teórica. Facultad de Ciencias \\ Universidad de Zaragoza. 50005 Zaragoza. Spain \\ E-mail: asorey@unizar.es,alessandro.santagatadunizar.es
}

\begin{abstract}
We analyze the transition of QCD effective potential from the Coulomb regime to the confinement regime in the heavy quark approximation. The instability of Coulomb potential in that approximation can be derived from first principles which confirms the Gribov picture of quark confinement. The transition is made possible by the breaking of conformal symmetry induced by the heavy quarks which allows the transition from a asymptotic freedom regime to a quark confinement regime. We find the critical distance between a quark anti-quark pair where the transition between the two regimes occurs for a small enough value of the strong coupling. This is in contrast with what happens in some supersymmetric theories where the phenomenon does not occur. The results point towards a confinement mechanism driven by thick strings rather than by fundamental strings.
\end{abstract}

Xth Quark Confinement and the Hadron Spectrum,

October 8-12, 2012

TUM Campus Garching, Munich, Germany

${ }^{*}$ Speaker. 


\section{Intoduction}

The most popular picture of confinement is provided by the dual superconductor scenario. In that picture the QCD vacuum behaves like a dual superconductor generated by the condensation of chromo-magnetic monopoles where the chromo-electric flux is expelled by the dual Meissner effect [1][2]. The immersion of a heavy quark-antiquark pair in such a magnetic superconducting vacuum generates a concentration of the chromo-electric flux lines along the segment connecting the two particles, which induces an effective quark-antiquark potential growing linearly with the distance. At large distances between a pair of quark-antiquark the flux tube behaves like a string which leads to quark confinement. This picture has been numerically confirmed but there is no analytic proof from first principles. Only in $N=2$ supersymmetric Yang-Mills theories an analysis carried out by Seiberg and Witten suggests that it could be the real confinement mechanism, but those models are not close to real QCD [3].

An alternative picture for confinement was suggested by Gribov in the early nineties [4][5]. Motivated by the instability of relativistic hydrogenoid atoms with $Z>137$ Gribov raised the possibility of a QCD vacuum instability due to the very large values that the effective $\alpha_{s}$ coupling constant can reach at the infrared regime. The instability would imply a vacuum decay on light quark-antiquark pairs [6]-[7]. From the Dyson-Schwinger equations of the light quark Green function Gribov found a critical value of $\alpha_{s}$

$$
\alpha_{\text {crit }}=\frac{2 N \pi}{N^{2}-1}\left(1-\sqrt{\frac{2}{3}}\right)
$$

beyond which $\alpha_{s}>\alpha_{\text {crit }}$ the theory becomes unstable [6]-[9].

In this note we shall show that the Gribov picture holds also for heavy quarks. To obtain some analytic understanding of the mechanism of quark confinement in real QCD we propose a new semi-classical approach, for SU(2) Yang-Mills theory coupled to a static heavy quark. A trivial classical solution of the equations of motion is represented by the Coulomb potential, however we shall show that this potential is not stable for couplings larger than a critical value $\alpha_{c}=\sqrt{5} / 2$. A similar critical value was found in earlier analyses [10]-[21] of Yang-Mills equations in a Coulomb background. However, our approach is based on the Euclidean path integral approach which permits the comparison with numerical results of the lattice gauge theories approach. Our main result is the discovery in that regime of a critical distance between a heavy quark-antiquark pair beyond which the Coulomb phase becomes unstable, while asymptotic freedom is preserved a shorter distances.

\section{Heavy Quark Coulomb Instability}

In presence of a static heavy quark theYang-Mills action is given by

$$
S^{Y M}(A)=-\frac{1}{2 g^{2}} \int d^{4} x \operatorname{Tr}\left(F^{\mu v} F_{\mu v}\right)+Q \int d x^{0} A_{0}^{3}(0),
$$

where for simplicity the quark color has been chosen to have only projection on the third component of Gell-Mann matrices. The quantum theory is encoded by the Euclidean functional integral which 
is dominated by the static critical configurations of the Euclidean action, i.e. Coulomb solutions of Euclidean Yang-Mills equations

$$
\vec{A}=0, \quad A_{0}^{3}(x)=i \frac{g^{2} Q}{4 \pi|\vec{x}|}=i \frac{\alpha}{|\vec{x}|}, \quad \alpha=\frac{g^{2} Q}{4 \pi} .
$$

Coulomb gauge fields are imaginary because we are in the Euclidean formalism. They should be considered as saddle points which dominate the functional integral outside the physical real range of gauge fields. The Gaussian approximation around these Coulomb backgrounds is given by the second order variation of the Euclidean action

$$
\delta^{2} S=-\int d^{4} x \operatorname{Tr} \tau^{\mu}\left(-\delta_{\mu v} D^{2}+D_{\mu} D_{v}-2\left[F_{\mu v} \cdot\right]\right) \tau^{\nu} .
$$

If the second order differential operator involved in (2.3) is positive the functional integral reduces to the inverse square root of its determinant. However, if the operator is non-positive their negative eigenvectors will give rise to vacuum instabilities. Having in mind the dual superconductor picture of QCD vacuum the search for vacuum instabilities can be restricted to pure static magnetic gauge field perturbations

$$
\vec{\tau}(x)=\frac{\vec{x} \times \vec{n}}{|\vec{x}|} \phi(\vec{x}) \mathrm{T}_{12}, \quad \tau_{0}=0,
$$

with negative eigenvalues of the second order variation operator

$$
\left(-\delta_{\mu v} D^{2}+D_{\mu} D_{v}-2\left[F_{\mu \nu}, \cdot\right]\right) \tau^{v}=-\lambda^{2} \tau_{\mu}
$$

where $\overrightarrow{\mathrm{n}}$ is any unit vector and $\mathrm{T}_{12}$ is any normalized linear combination of the first two components of Gell-Mann matrices $\left(\mathrm{T}_{12}^{2}=-1 / 4\right)$.

In spherical coordinates if we assume that $\phi(\vec{x})=\phi(r)$ with $r=|\vec{x}|$ the eigenvalue equation (2.4) becomes

$$
\left(\frac{d^{2}}{d r^{2}}+\frac{2}{r} \frac{d}{d r}-\frac{2-\alpha^{2}}{r^{2}}\right) \phi(r)=\lambda^{2} \phi(r) .
$$

This equation is well know and presents three different regimes depending on the strength of the coupling constant $\alpha$ [22]. If $\alpha^{2}<\frac{5}{4}$ there is no solution of (2.5) vanishing on the quark and in the absence of negative eigenvalues the Gaussian integral is convergent and the system is stable. If $\frac{5}{4}<\alpha^{2}<\frac{9}{4}$ there is one solution of (2.5) with $\lambda_{v}=2 \Lambda\left(\frac{\Gamma(1+v)}{\Gamma(1-v)}\right)^{\frac{1}{2 v}}$ and

$$
\phi(r)=\frac{K_{v}\left(\lambda_{v} r\right)}{\sqrt{\lambda_{v} r}}
$$

satisfying the boundary condition

$$
\lim _{r \rightarrow 0} 2 r \phi^{\prime}(r)=\lim _{r \rightarrow 0}(-1+2 v \operatorname{coth}[v \log (\Lambda r)]) \phi(r),
$$

where $v=\sqrt{9 / 4-\alpha^{2}}$. The arbitrary parameter $\Lambda$ introduced by the boundary condition (2.7) in order to guarantee the hermiticity of the second order variation operator of the Euclidean action breaks conformal invariance and is crucial for the existence of the negative eigenvalue

$$
-\lambda_{v}^{2}=-4 \Lambda^{2}\left(\frac{\Gamma(1+v)}{\Gamma(1-v)}\right)^{\frac{1}{v}} .
$$


If $\alpha^{2}>\frac{9}{4}$ there is an infinity of solutions of (2.5)

$$
\phi_{n}(r)=\frac{K_{v}\left(\lambda_{n} r\right)}{\sqrt{\lambda_{n} r}}
$$

with

$$
\lambda_{n}=2 \Lambda \exp \left(\frac{i \pi n}{v}+\frac{1}{2 v} \log \frac{\Gamma(1+v)}{\Gamma(1-v)}\right)
$$

satisfying the boundary condition

$$
\lim _{r \rightarrow 0}(-1+2 i v \cot [i v \log (\Lambda r)]) \phi_{n}(r)=\lim _{r \rightarrow 0} 2 r \phi_{n}^{\prime}(r) .
$$

This is a case of extreme instability of the Coulomb solution for the heavy quark background and again the parameter $\Lambda$ introduced by the boundary condition (2.10) breaks conformal invariance, but not completely since a discrete conformal symmetry is preserved. The boundary condition and the spectrum are invariant under the discrete rescaling of $\Lambda \rightarrow \Lambda e^{2 \pi i / v}$ [25].

The instability of the Coulomb phase is intrinsically associated to the breaking of conformal symmetry. In perturbation theory this emerges from the renormalization of the coupling constant $\alpha$. In this picture it arises from the need of fixing the boundary conditions of the singularity of quark potentials. The novelty is that in this case it implies the instability of the Coulomb vacuum background for large enough coupling constant. However, the connection of the picture with real confinement is not yet clear because one quark background alone does not match the global gauge invariance conditions of Gauss law. For this reason is convenient to analyze what happens with several quarks and their interactions.

\section{Quark-Antiquark Coulomb Instability}

In the presence of a pair of heavy quark-antiquark the instability of Coulomb phase should persist at large quark-antiquark distances but it is not clear whether or not it holds at all distances. The distance between the two quarks introduces a new scale in the theory which might affect the stability behavior.

In this case the Coulomb background solution of Euclidean Yang-Mills equations reads

$$
A_{0}^{3}(\vec{x})=\frac{\alpha}{\left|\vec{x}-L \vec{e}_{3}\right|}-\frac{\alpha}{\left|\vec{x}+L \vec{e}_{3}\right|} .
$$

Possible unstabilities can arise from static magnetic field perturbations of the form

$$
\vec{\tau}(x)=\frac{\vec{x} \times \overrightarrow{\mathrm{e}}_{3}}{\rho} \phi(\rho, z) \mathrm{T}_{12}, \quad \tau_{0}=0,
$$

where $\phi(\rho, z)$ satisfies the eigenvalue equation

$$
\left(\frac{\partial^{2}}{\partial \rho^{2}}+\frac{\partial^{2}}{\partial z^{2}}+\frac{1}{\rho} \frac{\partial}{\partial \rho}-\frac{1}{\rho^{2}}+\alpha^{2}\left(\frac{1}{\sqrt{\rho^{2}+(z-L)^{2}}}-\frac{1}{\sqrt{\rho^{2}+(z+L)^{2}}}\right)^{2}\right) \phi(\rho, z)=\lambda^{2} \phi(\rho, z)
$$

in cylindric coordinates. If we consider the same boundary conditions introduced in the previous section for both quarks we have the same three regimes of the coupling constant $\alpha$. The behavior 


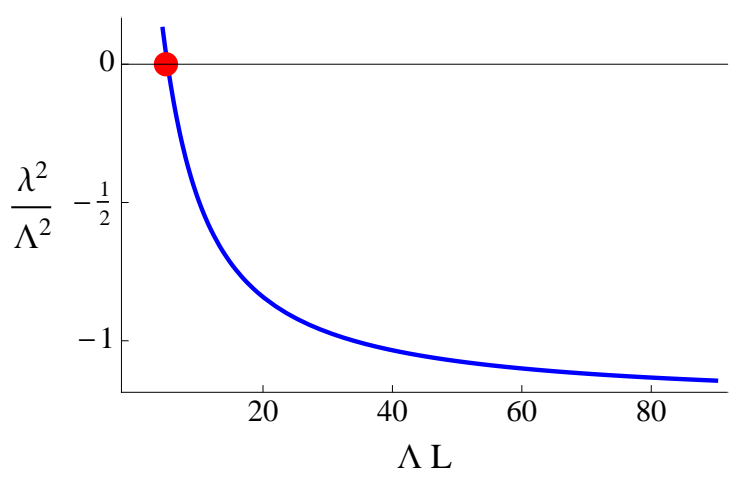

Figure 1: Unstable modes disappear at short $q-\bar{q}$ distances

in the three regimes is similar to that of only one quark but with some relevant differences. If $\alpha^{2}<\frac{5}{4}$ there is no solution of (3.2) vanishing at the quark and antiquark positions. The absence of negative eigenvalues implies again the stability of the Coulomb regime. If $\frac{5}{4}<\alpha^{2}<\frac{9}{4}$ there are two solutions $\phi_{ \pm}$of (3.2) satisfying the boundary condition (2.7). One solution $\phi_{+}$is even and the other $\phi_{-}$odd under z-reflection. Although the system is not invariant under the interchange of quark and anti-quark, the second order variation operator in (3.1) in invariant under this interchange.

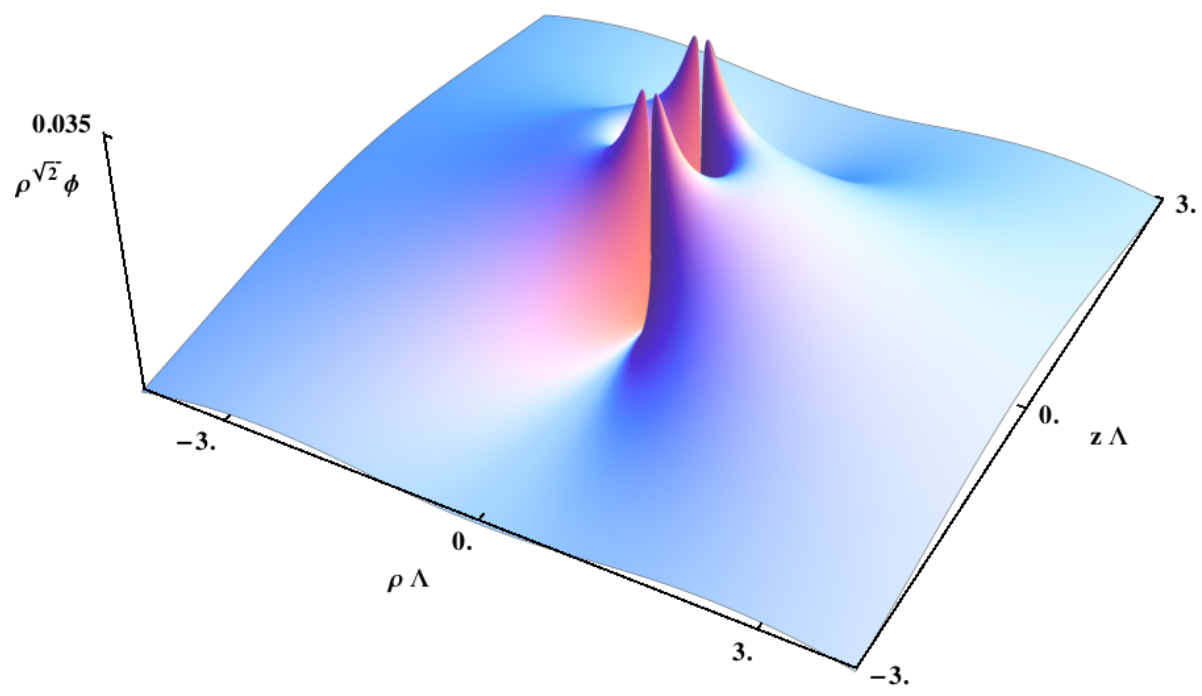

Figure 2: Unstable parity even mode of a heavy quark-antiquark system for $\alpha=\sqrt{17 / 8}$. The thick string connecting the two quarks suggests a confinement mechanism driven by thick strings rather than fundamental strings.

A novel interesting property is that for $2<\alpha^{2}<\frac{9}{4}$ these two negative eigenvalues disappear at short distances. There is a critical value $2 L_{c}$ of the distance between the two quarks where the parity even negative gauge field perturbation becomes a zero mode (see Fig. 1). This critical value marks the stability limit of Coulomb regime. The parity odd negative eigenvalue disappears at 
larger distances. Although the solutions are not analytic we can show numerically that they are essentially concentrated near the two quarks (see Fig. 2 and Fig. 3). The dependence of the critical distance $2 L_{c}$ with the coupling constant is also displayed in Fig. 4.

This result means that in this regime the Coulomb background provides a good description of the system a short distances $L<L_{c}$. If one includes one loop corrections we get asymptotic freedom in this regime. However, for $L>L_{c}$ the Coulomb background field is not stable and one expects to enter in the realm of confinement. Moreover, the lowest unstable mode (Fig. 2) exhibits a prominent thick string connecting the two quarks, supporting a picture of QCD where confinement is likely to be induced by thick strings rather than by fundamental strings [26, 27].

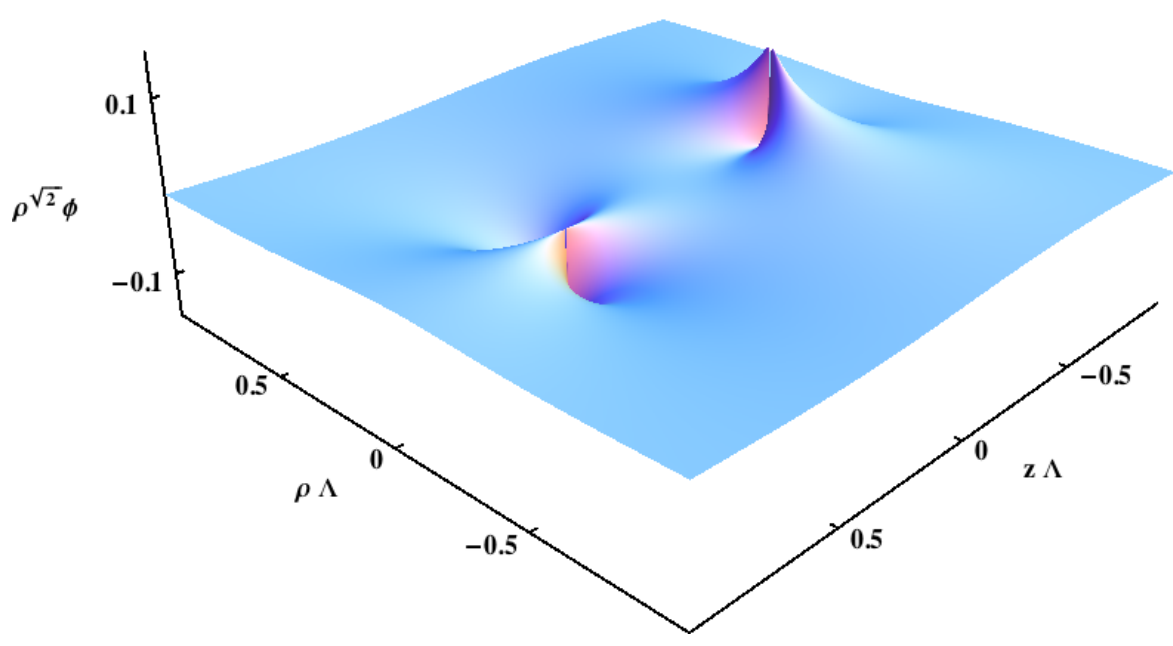

Figure 3: Unstable parity odd mode of a heavy quark-antiquark system for $\alpha=\sqrt{17 / 8}$

In the strong coupling regime $\alpha^{2}>\frac{9}{4}$ there is an infinity of solutions of (3.2) for any non-null distance $2 L>0$ between the quarks. All these unstable modes disappear as the quarks get closer and closer but for any finite distance the number of unstable modes is always infinite.

The above instabilities do not mean that the functional integral is not well defined. In fact the imaginary character of the Coulomb background field remind us that this is a saddle point of the Euclidean action. Choosing an integration contour locally orthogonal to the negative modes in the complex plane the Gaussian integral gives a positive finite contribution. However, the instability persists due to the fact that the quantum Hamiltonian of the field theory in such a background in not unitary. The above instability modes reappear in the form of time dependent zero modes with complex energies [28].

\section{Conclusions}

The above analysis provides new evidences showing that the Gribov's picture of confinement can be derived from first principles in the heavy quark approximation. The semiclassical expansion of the Euclidean functional integral around the Coulomb gauge field background is unstable for gauge coupling larger than some critical value $\alpha>\alpha_{c}=\sqrt{5} / 2$. The critical value of $\alpha$ know 


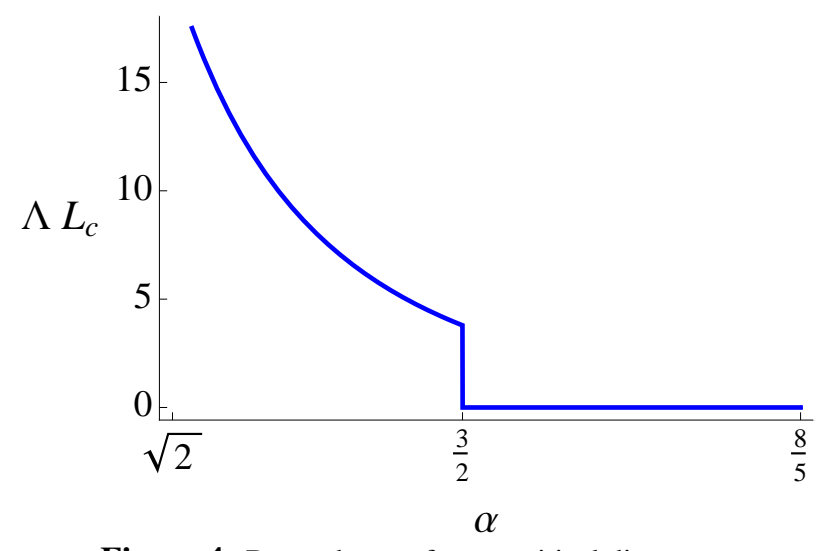

Figure 4: Dependence of $\mathrm{q}-\overline{\mathrm{q}}$ critical distance on $\alpha$

since the seventies [10]-[21] from a Minkowskian analysis of Yang-Mills equations in a Coulomb background is slightly higher $\alpha_{c}^{\prime}=3 / 2$. Our analysis is different but the nature of the singular problem is the same, which explains why the results are so similar. From a fundamental point of view our result, obtained in the Euclidean functional integral framework, is closer to the lattice approach were the numerical results provide complete evidence of quark confinement.

It is interesting to note that this critical value of the strong coupling constant on the presence of heavy quarks is of the same order of magnitude as the Gribov's critical value $\alpha_{\text {crit }}$ (1.1) for light quarks [4]. This provides another consistency check of Gribov's picture of confinement.

Another interesting feature of this approach is the mechanism of conformal symmetry breaking. In this case the symmetry is broken by the need of a boundary condition (2.7) in order to guarantee the hermiticity of the second order variation operator of the Euclidean action. The boundary condition introduces a dimensionfull parameter $\Lambda$ which breaks conformal invariance and generates unstable modes of the Euclidean action. On the other hand in this picture the compatibility of asymptotic freedom and confinement is explicit. Indeed, in the weak coupling regime $\sqrt{2}<\alpha<\frac{3}{2}$ there is a critical quark-antiquark distance $L_{c}$ such that Coulomb phase is stable for $L<L_{c}$ and instable for $L>L_{c}$. This implies that at short distances the quarks interact via Coulomb interaction which leads to asymptotic freedom once one takes into account only loop contributions to the renormalization of the coupling constant $\alpha$. However, at large distances $L>L_{c}$ their interaction is different which opens the possibility of a confining behavior. This proof of confinement requires further analysis but it can approached from this perspective [28].

The Coulomb phase instability does not appear in the supersymmetric case because there are the same negative modes for gauge fields that for the supersymmetric partners canceling out both pathological contributions to the Euclidean functional integral. This explains why supersymmetric theories can have a stable Coulomb phase at all distances [3].

Acknowledgements: We thank J.C. Cuchí, J. G. Esteve, J. Mateos Guilarte and G. Sierra for enlightening discussions on several aspects of singular potentials and confinement and specially to J. M. Muñoz-Casteñeda for collaboration in early stages of this work. We have been supported by the Spanish DGIID-DGA grant 2009-E24/2, the Spanish MICINN grants FPA2009-09638 and CPAN-CSD2007-00042. A. S. thanks DGIID-DGAfor a Ph. D. grant. 


\section{References}

[1] G. 't Hooft, in High Energy Physics, Ed. A. Zichichi, Ed. Compositori, Bologna (1976); Nucl. Phys. B 190 [FS3] (1981) 455.

[2] S. Mandelstam, Phys. Rep. 23 (1976) 245; Phys. Rep. 67 (1980) 109.

[3] N. Seiberg and E. Witten, Nucl. Phys. B431 (1994) 484.

[4] V.N. Gribov, Talk at Perturbative QCD Workshop, Lund, Sweden, May 21Đ24, 1991. Preprint LU-TP- 91-7 (unpublished).

[5] V.N. Gribov, Orsay lectures on confinement LPTHE-ORSAY 92-60 (1992) [Arxiv preprint: hep-ph/9403218]; LPTHE-ORSAY 94-20 (1994) [Arxiv preprint: hep-ph/9404332] and LPT-ORSAY 99-37 (1999) [Arxiv preprint: hep-ph/9905285].

[6] V.N. Gribov, Eur. Phys. J. C10 (1999)70.

[7] V.N. Gribov, Gauge Theories and Quark Confinement. Collection of works, Phasis Publishing House, Moscow (2002), 357.

[8] C. Ewerz, Eur. Phys. J. C13 (2000) 503 [arXiv:hep-ph/0001038].

[9] Yu.L. Dokshitzer and D.E. Kharzeev, Ann. Rev. Nucl. Part. Sci. 54, (2004) 487.

[10] J.E. Mandula, Phys. Rev. D 14 (1976) 3497; Phys. Lett. B 67 (1977) 175; Phys. Lett. B 69 (1977) 495.

[11] M. Magg, Phys. Lett. B 74 (1978) 246; Phys. Lett. B 77 (1978) 199; Phys. Lett. B 78 (1978) 481; Nucl. Phys. B 158 (1979) 154.

[12] J.E. Mandula and L. McLerran, Phys. Lett. B 73 (1978) 193.

[13] A.J.G. Hey and J.E. Mandula, Phys. Rev. D 19 (1979) 1856.

[14] P. Sikivie and N. Weiss, Phys. Rev. Lett. 40 (1978) 1411; Phys. Rev. D18 (1978) 3809; Phys. Rev. D 20 (1979) 487.

[15] P. Sikivie, Phys. Rev. D 20 (1979) 877.

[16] K.E. Cahill, Phys. Rev. Lett. 41 (1978) 599.

[17] I.B. Khriplovich, Sov. Phys. JETP 47 (1978) 18, Zh. Eksp. Teor. Fiz. 74 (1978) 37.

[18] R.A. Freedman, L. Wilets, S.D. Ellis and E.M. Henley, Phys. Rev. D 22 (1980) 3128.

[19] C.H. Oh, R. Teh and W.K. Koo, Phys. Lett. B 101 (1981) 337.

[20] L.J. Carson, R. Goldflam and L. Wilets, Phys. Rev. D 28 (1983) 385.

[21] L.J. Carson, Phys. Rev. D 29 (1984) 2355.

[22] K. M. Case, Phys. Rev. 80, (1950) 797.

[23] W. Frank, D.J. Land and R.M. Spector, Rev. Mod. Phys. 43 (1971) 36.

[24] K.S. Gupta and S.G. Rajeev, Phys. Rev. D48 (1993) 5940.

[25] M. Asorey, J.G. Esteve, A. Santagata and G. Sierra, In preparation.

[26] J. Polchinski and L. Susskind, String theory and the size of hadrons [arXiv: hep-th/0112204].

[27] V. Vyas, Intrinsic Thickness of QCD Flux-Tubes [arXiv: 1004.2679].

[28] M. Asorey and A. Santagata, In preparation. 